\title{
Safety and reliability of external fixation for basicervical and intertrochanteric fractures in high-risk elderly patients
}

\author{
George W. Boghdady $\cdot$ Mohammed Shalaby
}

Received: 22 September 2007 / Accepted: 24 November 2007/Published online: 5 December 2007

(C) Springer-Verlag 2007

\begin{abstract}
Forty elderly patients with basicervical and pertrochanteric fractures were managed with uniplanar $\mathrm{AO}$ external fixator under regional anaesthetic block of the femoral nerve and lateral cutaneous nerve of the thigh from April 2003 to March 2006. The mean age of the patients was $67.9 \pm 5.5$ years. External fixator application was performed under radiological control after closed reduction had been obtained. Comorbid factors, duration of surgery, duration of hospitalisation, complications, walking ability, time to union and mortality rate were recorded. Patients were followed up for a mean period of $12 \pm 4.5$ months. Superficial pin tract infection occurred in 13 patients, healing in varus $>10^{\circ}$ and with shortening $>2 \mathrm{~cm}$ occurred in six patients, and one patient suffered a spontaneous ipsilateral femoral neck fracture after removal of the fixator. The mean time for union was $10.4 \pm 1.2$ weeks. Rapid union rate and minor complications obtained in the present study are comparable to those obtained with standard internal fixation techniques. Minimal intraoperative blood loss, short operative time and early patient mobilisation are advantages signifying uniplanar external fixator application under regional anaesthetic block to be a viable option in
\end{abstract}

This work received no grant support in any form. This work has been done at Mansoura University Hospitals, Mansoura University, Dakahlia, Egypt.

G. W. Boghdady $(\bowtie)$

Orthopaedic Surgery and Traumatology,

Orthopaedic Department, Faculty of Medicine,

Mansoura University, PO Box 2, Mansoura 35516, Egypt

e-mail: georgewadeed@gmail.com

M. Shalaby

Anaesthesiology Department, Mansoura University Hospitals,

Mansoura, Egypt treatment of basicervical and pertrochanteric fractures in high-risk elderly patients.

Keywords Hip fractures - External fixators .

Nerve block · Frail elderly

\section{Introduction}

In developing countries, many patients with pertrochanteric fractures are unsuitable for treatment by conservative means or by conventional open reduction and internal fixation [4]. Moreover, the treatment of basicervical fractures, as with very proximal pertrochanteric fractures or low-neck fractures, has been controversial. Biomechanical studies suggest that basicervical fractures may be best treated similarly to pertrochanteric fractures rather than to neck fractures $[1,6,19]$. For the elderly patients the treatment of choice for pertrochanteric or basicervical fractures is surgical because non-operative treatment involves high morbidity and mortality compared to surgical treatment $[8,15,16,24]$. Moreover, the general condition of these patients is often poor due to the accompanying cardio-vascular, pulmonary, and in Egypt, liver diseases. In the majority of geriatric patients, walking ability is limited before the fracture, and co-existing anaemia and malnutrition make significant intra-operative blood loss intolerable [13]. A surgical procedure that achieves minimal surgical blood loss, short operative and hospitalisation time, minimal anaesthetic risk, the least morbidity and mortality rates, and early weight bearing is favoured [20]. This study was conducted to assess using the AO tubular external fixator in the management of both pertrochanteric and basicervical fractures in high-risk elderly patients. 


\section{Materials and methods}

Between April 2003 and March 2006, 40 elderly patients with basicervical fractures (13 patients) or pertrochanteric fractures (27 patients), were treated using an AO tubular uniplanar external fixator. There were 22 females and 18 males, with a mean age of $67.9 \pm 5.5$ years. The selection of the patients for the procedure was based on a poor state of general health producing a moderate or high surgical and anaesthetic risk in prolonged or invasive surgery. To assess the role of the severity of a patient's health problems at the time of admission, the rating system of the American Society of Anaesthesiologists (ASA) was used [24]. Table 1 summarizes the preoperative medical status and the ASA grade of all patients that necessitated choosing a minimally invasive technique. In addition, 9 of the 40 patients were of $\mathrm{Rh}$ negative blood group, for whom blood supplies were not available. The fractures were classified using AO classification and Jensen's modification of Evans' classification [10,18]. Nine patients were classified as $31-\mathrm{A} 1$ fractures (Fig. 1), and 31 patients as $31-\mathrm{A} 2$ according to AO classification (Figs. 2, 3). Fractures with subtrochanteric extension, and pathological fractures were excluded. Also, patients with associated fractures that might interfere with rehabilitation were also excluded. All patients were operated under regional anaesthesia through femoral nerve and lateral cutaneous nerve of thigh blocks.

A closed reduction of the fracture was performed under image intensification in both the anteroposterior (AP) and lateral planes before application of the fixator. Patients were placed supine on an orthopaedic table and closed reduction was usually accomplished by applying traction and internal rotation to the slightly adducted injured limb. The reduction was considered anatomical if the neck-shaft

Table 1 Co-morbid factors and ASA grade of patients included in the present study

\begin{tabular}{lll}
\hline Accompanying disease & No. of patients & Percentage \\
\hline Cardiovascular diseases & 33 & 82.5 \\
Uncontrolled diabetes mellitus & 28 & 70 \\
Respiratory diseases & 16 & 40 \\
Liver disease & 32 & 80 \\
Severe anaemia (Hb $\leq 9 \mathrm{mg} \%)$ & 35 & 87.5 \\
Others & & \\
Hypersplenism & 2 & 5 \\
Unsuitable skin condition & 2 & 5 \\
ASA grade & & \\
Grade II & 8 & 20 \\
Grade III & 26 & 65 \\
Grade IV & 6 & 15 \\
\hline
\end{tabular}

angle was between $120^{\circ}$ and $140^{\circ}$ and the distraction at the fracture site was less than $2 \mathrm{~mm}$. Minor valgus angulation $\left(<10^{\circ}\right)$ and a distraction of less than $5 \mathrm{~mm}$ were considered acceptable if an anatomical reduction could not be obtained. The fixator was applied using the AO principles and technique [5]. Two Schanz screws were inserted through stab incisions, from the base of the greater trochanter, across the fracture site into the femoral head. Another three Schanz screws were inserted at right angles into the middle half of the femoral shaft. Clamps were tightened and the final position was checked radiologically. On the first postoperative day, the patients started sitting on a bed or a chair, and from the second postoperative day, walking on a walker frame was started allowing full weight bearing as tolerated by the patient [14]. Daily care of the Schanz screws and the surrounding skin using povidone iodine solution was applied meticulously. All patients were followed up for a mean of $12 \pm 4.5$ months. The patients were evaluated clinically and radiologically, bi-weekly for the first month, then monthly for the next 5 months, and subsequently every 6 months. The radiological evaluation of all patients was made with AP and lateral radiographs of the proximal femur. Clinical examination included range of motion of the hip, knee and limb-length discrepancy. Operative time, units of transfused blood, duration of hospitalisation, complications, post-operative walking ability, healing time, and mortality were recorded for a minimum of 6 months.

\section{Results}

The mean operative time was $40.9 \pm 8.8$ min ranging from 30 to $60 \mathrm{~min}$, including time for positioning, and closed reduction of the fracture, but excluding the time for regional anaesthesia. The mean actual time for fixator application was $21.9 \pm 5.1 \mathrm{~min}$ ranging from 15 to 35 minutes. The mean hospital stay was $3 \pm 0.9$ days ranging from 2 to 5 days. No intraoperative complications were encountered. There was no need for intraoperative blood transfusion in any patient because blood loss was negligible. Postoperatively 11 patients received 2-3 units for correction of anaemia (Table 2).

The mean time for union was $10.4 \pm 1.2$ weeks ranging from 9 to 13 weeks. External fixators were removed after a mean period of $13.3 \pm 1.5$ weeks ranging from 11 to 16 weeks. They were kept for 2-3 weeks after radiological union and then removed.

Seven patients were walking freely at their last follow up while 28 patients were walking with assisted means, and 5 patients suffered limited walking despite assisted means. The range of motion of the hip and knee joints were $90^{\circ}$ or more in all patients at their final follow up. Seventeen 
Fig. 1 Preoperative $(\mathbf{a}, \mathbf{b})$, immediate postoperative $(\mathbf{c}, \mathbf{d})$, and 15 months follow up (e) radiographs of 63-years-old patient with basicervical fracture (AO: 31-A1.1) showing united fracture in anatomical position with osteoporosis
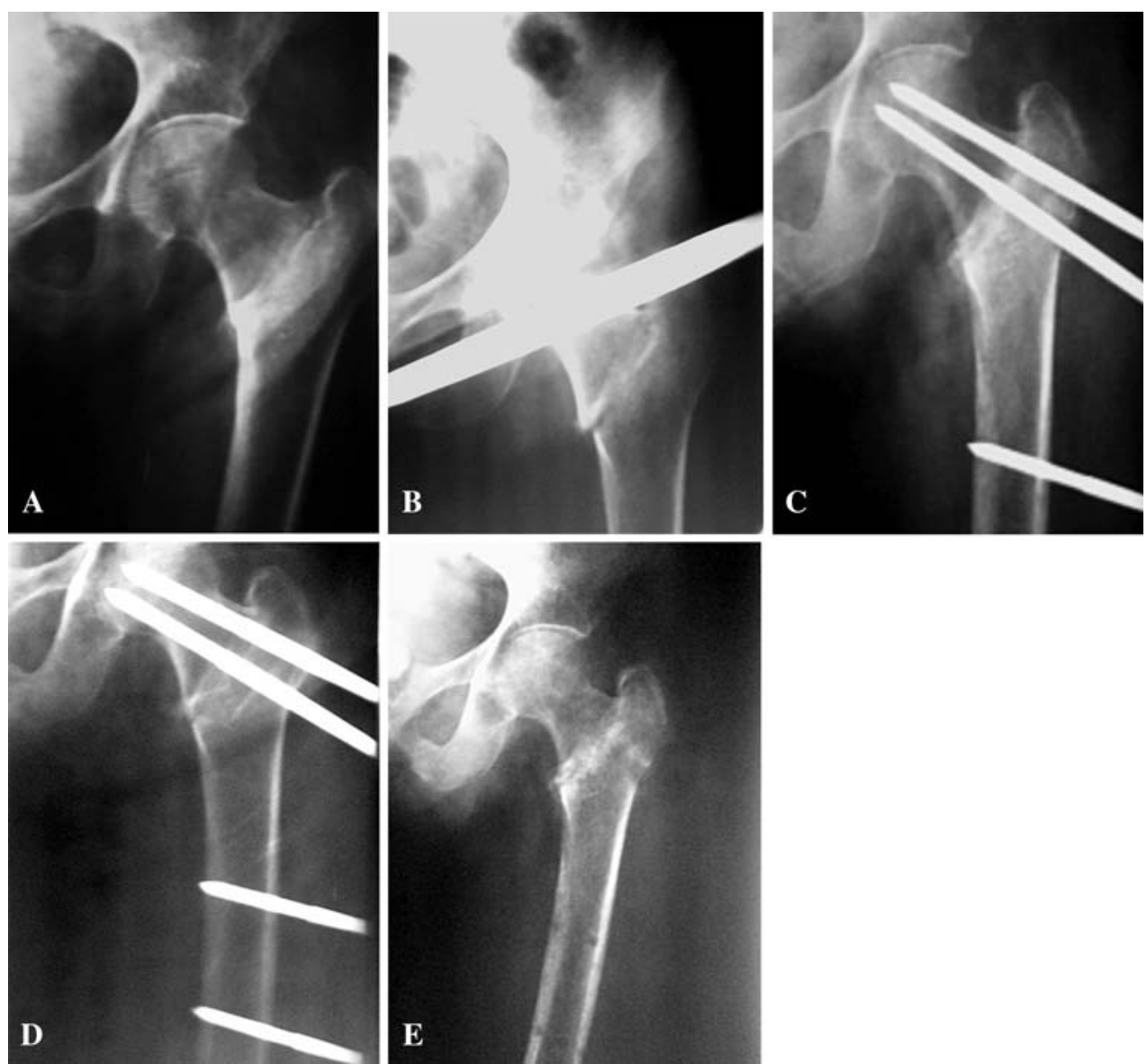

patients suffered quadriceps muscle wasting that accounted for inability to walk freely in these patients.

The most common postoperative complications (Table 3) were superficial pin tract infection in 13 patients, loosening of the Schanz screws in 3 patients, malunion in varus position $\left(>10^{\circ}\right)$ with shortening $>2 \mathrm{~cm}$ in 6 patients. One patient suffered a deep infection of the most cephalic Shanz screws, and one patient sustained spontaneous ipsilateral femoral neck fracture 3 weeks after removal of the fixator. There were no cardio-respiratory complications. Three patients developed urinary tract infections. No mortalities occurred in the perioperative period (within the first 30 days postoperatively), while seven patients died within 1 year postoperatively (all after a minimum of 6 months duration, and due to cardiac problems or liver cell failure).

\section{Discussion}

Open reduction and internal fixation of pertrochanteric fractures is a treatment 'gold standard', but the morbidity and mortality rates are high in elderly patients with significant co-morbid factors. Despite the differences in fracture stability, it is recommended that basicervical fractures are treated similarly to pertrochanteric fractures rather than to transcervical fractures [6,19]. Nonoperative treatment involves prolonged immobilisation and hospitalisation, with many concomitant problems and high mortality [3]. Obtaining a perfect reduction of the pertrochanteric fracture may not be the main objective for this high-risk group but to operate with the least blood loss in the shortest time and an anaesthetic technique of low risk, which ultimately facilitates early mobilisation in the postoperative period [20].

To avoid classic open surgery, many mini-invasive techniques have been introduced for treatment of such fractures, arguing that they offer better clinical outcome at no extra expense and with minimal complications [9, 21, 23]. Previous reports on external fixation showed short operating and hospitalisation time, high fracture union rates, and minimal surgical blood loss $[3,4,7,11,13,17$, $20,22]$. The present study was conducted using the minimally invasive technique of external fixation for the management of both pertrochanteric and basicervical fractures in elderly high-risk patients.

Many modifications concerning the external fixation technique have been introduced [3, 13, 17, 20, 22]. For 
Fig. 2 Preoperative $(\mathbf{a}, \mathbf{b})$, immediate postoperative $(\mathbf{c}, \mathbf{d})$, and 16 months follow up (e) radiographs of 65-years-old patient with three part trochanteric fracture (AO: 31A2.1) with solid union and restoration of normal neck shaft angle
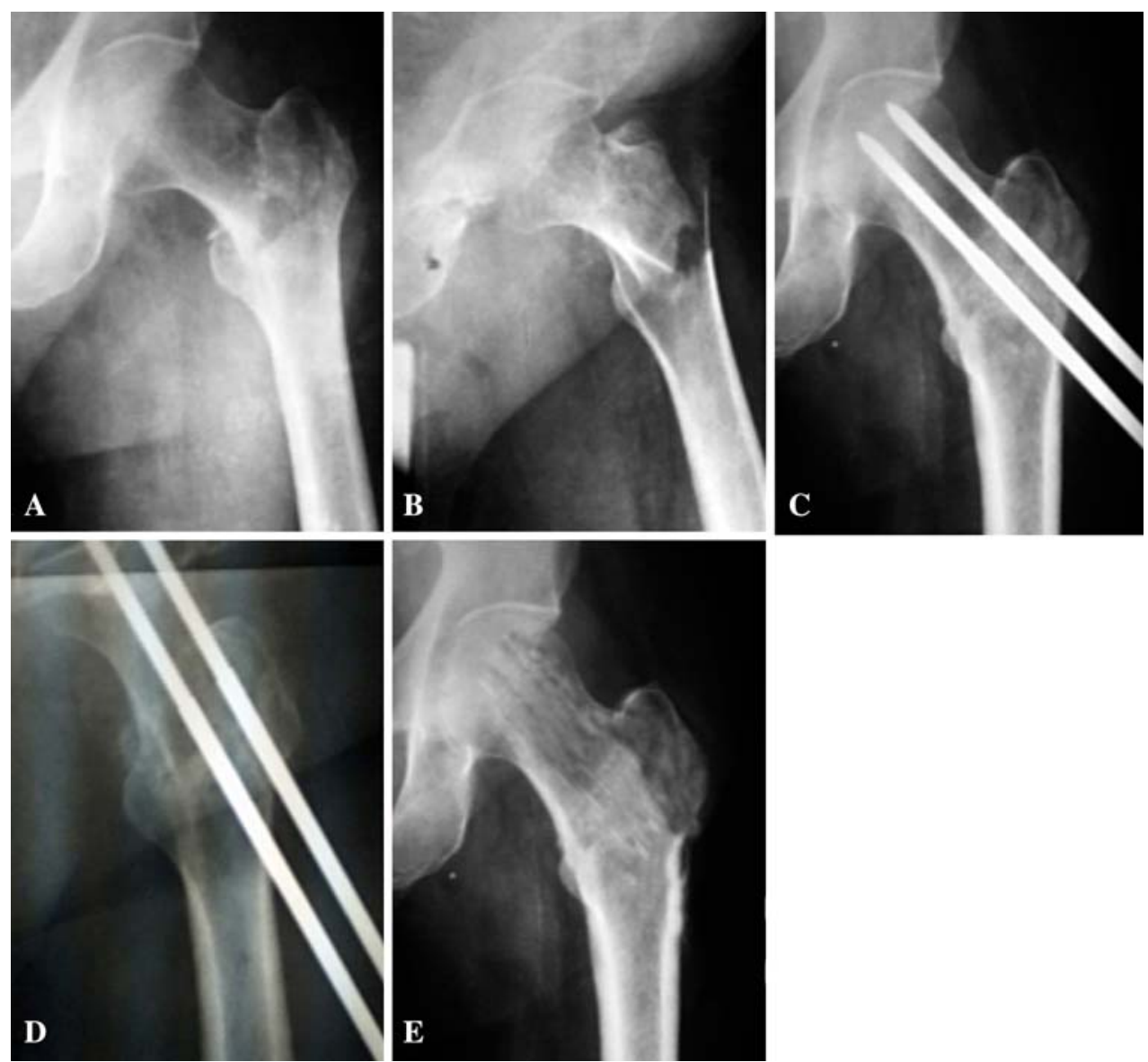

developing countries, most of these types of fixators are expensive and cannot be afforded by patients or hospitals. The reasonable cost, the availability, and familiarity with the AO tubular external fixator were the reasons for its use in the present study.

To further minimize the surgical risk on these vulnerable patients, surgeons choose to avoid general anaesthesia and opt for regional techniques $[3,4,7,13]$. Our experience with spinal or epidural anaesthesia in these patients is that it is usually difficult to adequately position a patient with such a proximal femoral fracture for such a technique. Local infiltration can be time consuming and not provide adequate anaesthesia for surgery. For the aforementioned reasons, anaesthetic block of both femoral nerve and lateral cutaneous nerve of the thigh was the technique used in the present study. The technique was successful in all patients, which allowed for closed reduction of the fracture, easy application of the fixator, and a reasonable period of postoperative analgesia.

The mean operative time for fixator application in the present study was $21.9 \pm 5.1 \mathrm{~min}$, which was similar to times recorded by Kourtzis et al. [13] and Vossinakis et al. [22]; and less than those recorded by Tomak et al. [20] and Christodoulou et al. [3]. Familiarity with the application technique and the few instruments needed for the AO external fixator application played a significant role in reducing the mean operative time. As was reported in previous studies $[3,4,7,11,13,20,22]$ no intraoperative complications occurred and there was no need for intraoperative blood transfusion as the blood loss was negligible.

The mean hospitalisation period in the present study ( $3 \pm 0.9$ days) was less than those recorded by Christodoulou et al. [3] (6 days), Kourtzis et al. [13] (7.2 days), Tomak et al. [20] (7.8 days), and Vossinakis et al. [22] ( 8 days). In this study, the choice of stabilisation by external fixed was made on admission. The patient was discharged as soon as ambulation with a walker was deemed safe and care of fixator pin-sites had been taught.

The meantime needed to achieve union in the present study (10.4 \pm 1.2 weeks) is similar to that reported by Ozdemir et al. [17], but less than that reported by other studies $[4,11,20](12-16$ weeks). The mean age of patients in the present study is lower than those of the three aforementioned studies. In addition, patients in the present study were encouraged to weight-bear fully as early as possible. This may account for the rapid union rate achieved. 
Fig. 3 Preoperative (a, b), immediate postoperative $(\mathbf{c}, \mathbf{d})$, and 14 months follow up (e) radiographs of 71-years-old patient with three part trochanteric fracture (AO: 31 A2.2) with malunited fracture in varus (neck shaft angle of $110^{\circ}$ )
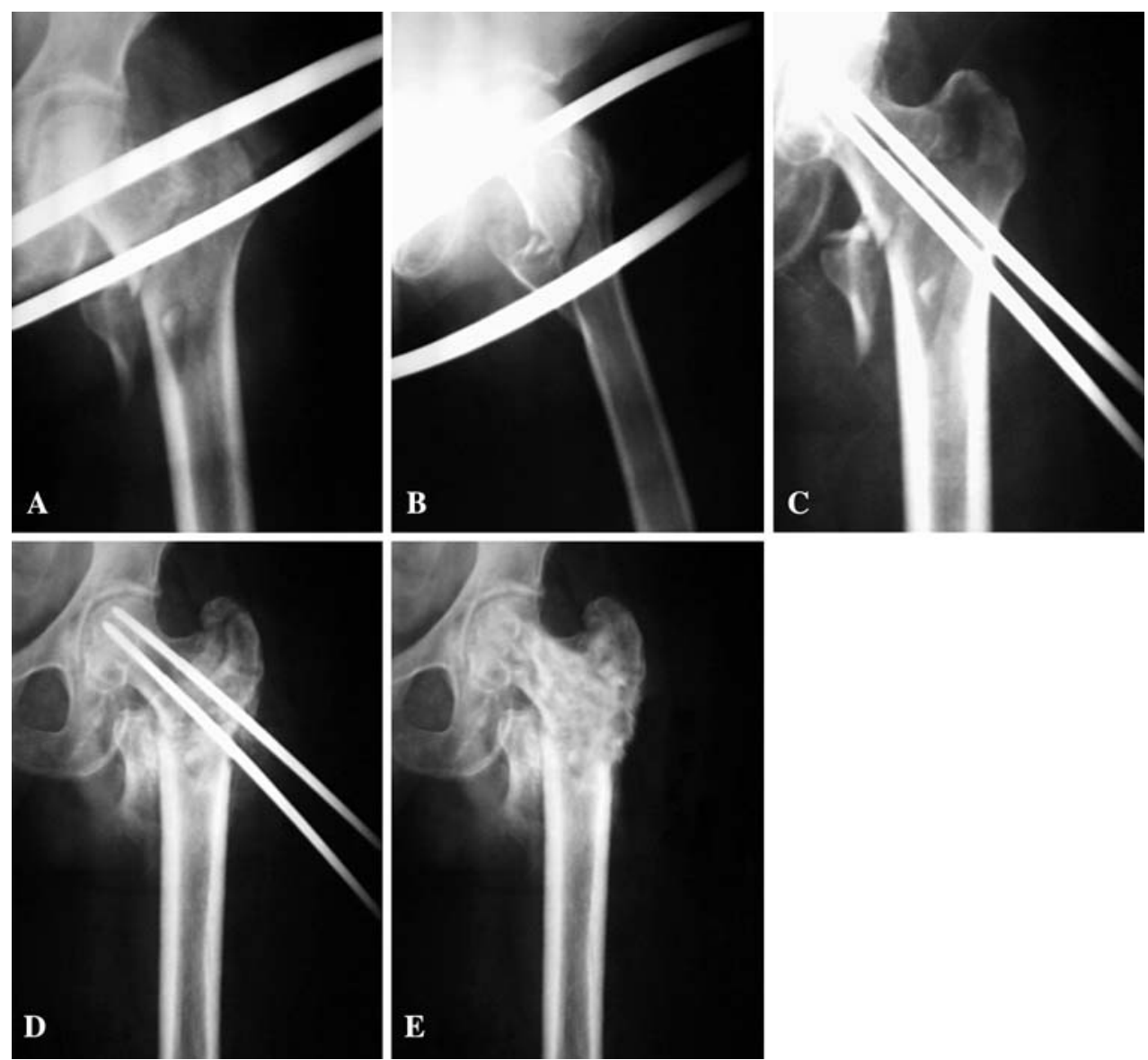

As with previous studies $[3,4,7,11-13,17,20,22]$, the most common complication in the present study was superficial pin tract infection occurring in 13 patients. Management by administration of oral antibiotics, application of a local antibiotic preparation and meticulous care of the skin around the pins was successful. Three patients showed radiological loosening of these pins at 6-7 weeks after surgery. Complete healing of the pin tracts occurred within 10 days after fixator removal and did not affect the final outcome of these patients. Another patient developed a deep pin tract infection that necessitated removal of the pin. Union was delayed to 13 weeks with varus of $15^{\circ}$ and shortening of $2.5 \mathrm{~cm}$. This patient walked with difficulty despite assisted means. However, the rate of deep infection occurring in the present study is less than that reported by other studies $[4,13,20]$.

The incidence of loss of reduction and varus malunion $>10^{\circ}$ with shortening $>2 \mathrm{~cm}$ in the present set of patients (6 patients) is comparable to that reported by many previous studies [3, 4, 11, 13, 17, 25]. All of these patients suffered unstable pertrochanteric fractures, five suffered superficial pin tract infections, and 1 suffered deep pin tract infection. The varus and shortening was explained by Dahl et al. [4] to be due to either fixation in varus or to collapse of the neck shaft angle on weight bearing. In this study, none of the fractures were fixed in varus. As a consequence, malreduction was not a factor producing shortening in the 6 patients but probably the type and pattern of fracture and its subsequent stability. However, Kourtzis et al. [13] suggested that the low demands of the elderly permit this degree of shortening to be of no significant functional compromise. None of the nine patients with basicervical fractures in the present study suffered this complication. To our knowledge, no previous studies have reported on the technique of external fixation in treatment of basicervical fractures.

One patient suffered a spontaneous fracture of the ipsilateral neck of femur after union of the pertrochanteric fracture and within 3 weeks of fixator removal. He was treated electively by hemiarthroplasty. Buciuto et al. [2] reported on seven patients with unstable pertrochanteric fractures fixed with either a fixed-angled plate or a DHS, who sustained spontaneous fractures of the femoral neck after removal of the implant. They stated that the mechanism of such complication is unknown. To our knowledge, no previous reports have recorded this complication with patients treated with an external fixator.

In contrast to Dobbs et al. [8], no deaths occurred within the first 30 postoperative days. The overall 1-year mortality 
Table 2 Patients' age, blood group, properative and postoperative $\mathrm{Hb} \%$

\begin{tabular}{|c|c|c|c|c|}
\hline $\begin{array}{l}\text { Patient } \\
\text { number }\end{array}$ & Age & $\begin{array}{l}\text { Blood } \\
\text { group }\end{array}$ & $\begin{array}{l}\text { Preoperative } \\
\text { Hb level (mg\%) }\end{array}$ & $\begin{array}{l}\text { Postoperative } \\
\text { Hb level (mg\%) }\end{array}$ \\
\hline 1 & 55.00 & $\mathrm{O}+\mathrm{ve}$ & 8.8 & 8.7 \\
\hline $2^{\mathrm{a}}$ & 60.00 & $B+v e$ & 8.5 & 8.4 \\
\hline 3 & 67.00 & $\mathrm{O}+\mathrm{ve}$ & 8.6 & 8.5 \\
\hline 4 & 64.00 & $A+v e$ & 9 & 8.6 \\
\hline 5 & 70.00 & $A-v e$ & 8.9 & 8.5 \\
\hline $6^{\mathrm{a}}$ & 69.00 & $\mathrm{AB}+\mathrm{ve}$ & 8 & 7.8 \\
\hline $7^{\mathrm{a}}$ & 73.00 & $B+v e$ & 7.5 & 7.4 \\
\hline 8 & 78.00 & $A+v e$ & 10.5 & 9.9 \\
\hline $9^{\mathrm{a}}$ & 77.00 & $\mathrm{O}-\mathrm{ve}$ & 6.5 & 6.2 \\
\hline $10^{\mathrm{a}}$ & 75.00 & $\mathrm{AB}+\mathrm{ve}$ & 8.5 & 8.1 \\
\hline 11 & 73.00 & $B+v e$ & 8.6 & 8.5 \\
\hline 12 & 68.00 & $B-v e$ & 9.2 & 9 \\
\hline 13 & 65.00 & $\mathrm{AB}+\mathrm{ve}$ & 9 & 8.6 \\
\hline 14 & 64.00 & $\mathrm{O}+\mathrm{ve}$ & 9 & 8.9 \\
\hline 15 & 59.00 & $A+v e$ & 8.8 & 8.7 \\
\hline 16 & 60.00 & $A+v e$ & 9 & 8.7 \\
\hline 17 & 62.00 & $B+v e$ & 9 & 8.7 \\
\hline 18 & 67.00 & $\mathrm{AB}+\mathrm{ve}$ & 8.6 & 8.4 \\
\hline 19 & 66.00 & $\mathrm{AB}-\mathrm{ve}$ & 9 & 8.6 \\
\hline 20 & 69.00 & $\mathrm{O}+\mathrm{ve}$ & 9 & 8.7 \\
\hline 21 & 70.00 & $B+v e$ & 13.6 & 13.4 \\
\hline 22 & 75.00 & $A-v e$ & 8.8 & 8.6 \\
\hline $23^{\mathrm{a}}$ & 71.00 & $A+v e$ & 9 & 8.5 \\
\hline $24^{\mathrm{a}}$ & 74.00 & $B+v e$ & 8.2 & 8 \\
\hline 25 & 65.00 & $A+v e$ & 8.8 & 8.5 \\
\hline 26 & 63.00 & $A+v e$ & 8.9 & 8.6 \\
\hline 27 & 59.00 & $\mathrm{O}-\mathrm{ve}$ & 8.9 & 8.4 \\
\hline 28 & 67.00 & $B+v e$ & 9 & 8.6 \\
\hline $29^{\mathrm{a}}$ & 68.00 & $\mathrm{AB}+\mathrm{ve}$ & 8 & 7.4 \\
\hline 30 & 69.00 & $A+v e$ & 8.8 & 8.4 \\
\hline 31 & 63.00 & $B-v e$ & 9 & 8.8 \\
\hline 32 & 65.00 & $A+v e$ & 8.5 & 8.4 \\
\hline $33^{\mathrm{a}}$ & 67.00 & $A+v e$ & 9 & 8.4 \\
\hline $34^{\mathrm{a}}$ & 68.00 & $B+v e$ & 8.8 & 8.6 \\
\hline 35 & 70.00 & $A-v e$ & 8.9 & 8.7 \\
\hline $36^{\mathrm{a}}$ & 76.00 & $\mathrm{O}+\mathrm{ve}$ & 8.5 & 8.1 \\
\hline 37 & 75.00 & $B-v e$ & 8.9 & 8.7 \\
\hline 38 & 74.00 & $\mathrm{O}+\mathrm{ve}$ & 9.8 & 9.6 \\
\hline 39 & 65.00 & $\mathrm{AB}+\mathrm{ve}$ & 8.5 & 8.3 \\
\hline 40 & 69.00 & $\mathrm{O}+\mathrm{ve}$ & 12.8 & 12.6 \\
\hline
\end{tabular}

\footnotetext{
${ }^{\text {a }}$ Patients who received postoperative blood transfusion to correct anaemia
}

rate is comparable to other studies [3, 13]. The mean age and the preexisting diseases account for the mortality in this patient group [8].
Table 3 General and local postoperative complications reported in the present study

\begin{tabular}{ll}
\hline Postoperative complications & No.of patients \\
\hline General complications & 0 \\
Pulmonary Embolism & 0 \\
Chest infections & 0 \\
Cardiac episode (myocardial & \\
$\quad$ infarction or heart failure & 0 \\
Liver cell failure & 0 \\
Renal insufficiency & 3 \\
Urinary tract infections & \\
Local complications & 13 \\
Superficial pin tract infection & 1 \\
Deep pin tract infection & 6 \\
Malunion with shortening & 3 \\
Loosening of the pins & 1 \\
Spontaneous femoral neck fracture & 0 \\
Migration of pins in the femoral head &
\end{tabular}

\section{Conclusion}

Within the limitations of the present study, negligible blood loss, rapid union rate, early mobilisation, and reasonable minor complications reported with the use of a uniplanar AO external fixator under regional anaesthetic block are comparable to those obtained with standard fixation techniques [12, 21, 22, 25]. It offers the major advantages of mini-invasive surgery and can allow fast and good functional recovery. The overall morbidity and mortality rates are reasonable enough to recommend this technique for the high-risk elderly patients.

\section{References}

1. Blair B, Koval KJ, Kummer F et al (1994) Basicervical fractures of the proximal femur. A biomechanical study of 3 internal fixation techniques. Clin Orthop Relat Res 306:256-263

2. Buciuto R, Hammer R, herder A (1997) Spontaneous subcapital femoral neck fracture after healed trochanteric fracture. Clin Orthop Relat Res 342:156-163

3. Christodoulou NA, Sdrenias CV (2000) External fixation of select intertrochanteric fractures with single hip screw. Clin Orthop Relat Res 381:204-211

4. Dahl A, Varghese M, Bhasin VB (1991) External fixation of intertrochanteric fractures of the femur. J Bone Joint Surg (Br) 73-B:955-958

5. Dell'Oca AF (2000) External fixation. In: Ruedi TP, Murphy WM (eds) AO principles of fracture managemant. George Thieme Verlag, Stuttgart, pp 233-247

6. Deneka DA, Simonian PT, Stankewich CJ et al (1997) Biomechanical comparison of internal fixation techniques for the treatment of unstable basicervical femoral neck fractures. J Orthop Trauma 11:337-343 
7. Devgan A, Sangwan SS (2002) External fixator in the management of trochanteric fractures in high risk geriatric patients. A friend to the elderly. Indian J Med Sci 56:385-390

8. Dobbs RE, Parvizi J, Lewallen DG (2005) Perioperative morbidity and 30-day mortality after intertrochanteric hip fractures treated with internal fixation or arthroplasty. J Arthroplasty 20:963-966

9. Herrera A, Domingo LJ, Calvo A et al (2002) A comparative study of trochanteric fractures treated with gamma nail or the proximal femoral nail. Int Orthop 26:365-369

10. Jin W-J, Dai L-Y, Cui Y-M et al (2005) Reliability of classification systems for intertrochanteric fractures of the proximal femur in experienced orthopaedic surgeons. Injury 35:858-861

11. Kamble KT, Murthy BS, Pal V et al (1996) External fixation in unstable intertrochanteric fractures of femur. Injury 27:139-142

12. Karn NK, singh GK, Kumar P et al (2006) Comparison between external fixation and sliding hip screw in the management of trochanteric fracture of the femurin Nepal. J Bone Joint Surg $(\mathrm{Br})$ 88-B: $1347-1350$

13. Kourtzis N, Pafilas D, kasimatis G (2001) Management of perttrochanteric fractures in the elderly patients with an external fixator. Injury 32:S-D-115-S-D-128

14. Koval KJ, Sala DA, Kummer FJ et al (1998) Postoperative weight-bearing after a fratcture of the femoral neck or an intertrochanteric fracture. J Bone Joint Surg (Am) 80:352-366

15. Koval KJ, Swiontowski MF (1999) Trauma: Hip. In: Beaty JH (ed) Orthopaedic knowledge update, vol 6. American Academy of Orthopaedic Surgeons, pp 441-453

16. Koval KJ, Zuckerman JD (1994) Hip fractures: evaluation and treatment of intertrochanteric fractures. J Am Acad Orthop Surg 2:150-156
17. Ozdemir H, Dabak TK, Urguden M et al (2003) A different treatment modality for trochanteric fractures of the femur in surgical high-risk patients: a clinical study of 44 patients with 21month follow-up. Arch Orthop Trauma Surg 123:538-543

18. Pervez H, Parker MJ, Pryor GA et al (2002) Classification of trochanteric fracture of the proximal femur: a study of the reliability of current systems. Injury 33:713-715

19. Su BW, Heyworth BE, Protopsaltis TS et al (2006) Basicervical versus intertrochanteric fractures: an analysis of radiographic and functional outcomes. Orthopedics 29:919-925

20. Tomak Y, Kacaoglu M, Piskin A et al (2005) Treatment of intertrochanteric fractures in geriatric patients with a modified external fixator. Injury 36:635-643

21. Utrilla AL, Reig Js, Munoz FM et al (2005) Trochanteric gamma nail and compression hip screw for trochanteric fractures. A randomized, prospective, comparative study in 210 elderly patients with a new design of the gamma nail. J Orthop Trauma 19:229-233

22. Vossinakis IC, Badras LS (2002) The external fixator compared with the sliding hip screw for pertrochanteric fractures of the femur. J Bone Joint Surg (Br) 84-B:23-29

23. Waters TS, Gibbs DMR, Dorrell JH et al (2006) Percutaneous dynamic hip screw. Injury 37:751-754

24. White BL, Fisher WD, Laurin CA (1987) Rate of mortality for elderly patients after fracture of the hip in the 1980's. J Bone Joint Surg (Am) 69-A:1335-1140

25. Moroni A, Faldini C, Pegreffi F et al (2005) Dynamic hip screw with external fixation for treatment of osteoporotic pertrochanteric fractures. A prospective randomized study. J Bone Joint Surg (Am) 87(4):753-759 